\title{
A Note on Hypoellipticity of Degenerate Elliptic Operators
}

Dedicated to Professor Mutsuhide Matsumura on his 60th birthday

By

\section{Minoru KolKE*}

This note concerns $C^{\infty}$ hypoellipticity for differential operators (in $\boldsymbol{R}^{3}$ ) of the form

$$
L=D_{t}^{2 m}+f(t) D_{x}^{2 m}+g(t) D_{y}^{2 m}, \quad D_{t}=-i \partial / \partial t, \quad m=1,2, \cdots .
$$

Here we assume
(A.1) 1) $f$ and $g$ belong to $C^{\infty}(-\delta, \delta)$ for some $\delta>0$,

2) $f(t)>0$ and $g(t)>0$ for $t \neq 0$.

Concerning operators closely related to $L$, criteria for the hypoellipticity have been recently given by several authors See Fedii [2], Hoshiro [4,5], Kusuoka and Stroock [6] and Morimoto [7, 8, 9, 10]. In particular, Hoshiro considered the same operator as $L$ with the assumptions (A.1) and

(A.2) both of $f$ and $g$ are non-decreasing in $[0, \delta)$, and non-increasing in $(-\delta, 0]$.

In this note we shall prove the following Theorems 1 and 2, which show that the condition

$$
\lim _{t \rightarrow 0} \mu(t ; g) \log f(t)=\lim _{t \rightarrow 0} \mu(t ; f) \log g(t)=0
$$

is equivalent to the hypoellipticity for $L$ under (A.1) and (A.2), where

$$
\begin{aligned}
\mu(t ; g) & =\max \left\{g(s)^{1 /(2 m)}|t-s| ; s \text { is between } 0 \text { and } t\right\} \\
& =\max \left\{g(\theta t)^{1 /(2 m)}(1-\theta)|t| ; 0 \leq \theta \leq 1\right\} .
\end{aligned}
$$

Theorem 1. If (A.1) holds, then (C) implies that $L$ is hypoelliptic (near

Communicated by S. Matsuura, March 22, 1991.

1991 Mathematics Subject Classification: 35H05 35J70

* Shibaura Institute of Technology, 307 Fukasaku, Omiya 330, Japan. 
$t=0$ )。

Theorem 2. Let (A.1) and (A.2) hold. Then (C) holds if L is hypoelliptic.

Notice that no assumption other than (A.1) is required in Theorem 1 whether $m=1$ or not (cf. Theorem 2 of [5] and Theorem 1.1 of [7]).

Example. Let $\sigma$ be a constant and

$$
L=D_{t}^{2 m}+e^{-2 m /|t|} D_{x}^{2 m}+\exp \left[-|t|^{-\sigma} e^{1 /|t|}\right] D_{y}^{2 m} \text {. }
$$

Then the Theorems show that $L$ is hypoelliptic if and only if $\sigma<2$.

We get the following Corollary at once (cf. Theorem 8.41 of [6], Theorem 3 of [8] and Proposition 1 of [10]).

Corollary. Let $f$ satisfy the conditions in (A.1). Then the condition

$$
\lim _{t \rightarrow 0} t \log f(t)=0
$$

implies that the operator

$$
D_{t}^{2 m}+f(t) D_{x}^{2 m}+D_{y}^{2 m}
$$

is hypoelliptic. If $f$ satisfies (A.2) in addition to (A.1), then the converse is also true.

We prove Theorems 1 and 2 in Sections 1 and 2, respectively. Our proofs are modifications of those in [4, 5]. We use the well known integral inequality of Hardy and an interpolation theorem in Sobolev spaces.

\section{§1. Proof of Theorem 1}

In view of Proposition 2 of [5], it suffices to prove the following: The condition

$$
\lim _{t \rightarrow 0} \mu(t ; g) \log f(t)=0
$$

together with (A.1) implies that, for every $\varepsilon_{0}>0$, there exists an $N\left(\varepsilon_{0}\right)>1$ such that

$$
(\log \xi)^{2 m} \int g(t)|\nu(t)|^{2} d t \leq \varepsilon_{0}\left[\int\left|\nu^{(m)}(t)\right|^{2} d t+\xi^{2 m} \int f(t)|\nu(t)|^{2} d t\right]
$$

for $\nu \in C_{0}^{m}(-\delta, \delta)$ and $\xi \geq N\left(\varepsilon_{0}\right)$.

We may assume $g$ and the derivatives of $f$ are bounded in $(-\delta, \delta)$ (by re- 
placing $\delta$ with a smaller one if necessary). Since (2) holds whenever $f(0)>0$, we only consider the case where $f(0)=0$.

We use the "sew together" method as in [4].

First, we have

\section{Lemma 1. The inequality}

$$
\int_{0}^{a} g(t)|\nu(t)|^{2} d t \leq 4 \mu(a ; g)^{2 m} \int\left|\nu^{(m)}(t)\right|^{2} d t
$$

holds if $0<a<\delta, \nu \in C_{0}^{m}(-\delta, \delta)$ and $\nu^{(k)}(a)=0(k=0, \cdots, m-1)$. Similarly, the estimate

$$
\int_{-b}^{0} g(t)|\nu(t)|^{2} d t \leq 4 \mu(-b ; g)^{2 m} \int\left|\nu^{(m)}(t)\right|^{2} d t
$$

holds if $0<b<\delta, \nu \in C_{0}^{m}(-\delta, \delta)$ and $\nu^{(k)}(-b)=0(k=0, \cdots, m-1)$.

Proof. We prove (3). Since

$$
\nu(t)=\int_{t}^{a} \frac{(-1)^{m}}{(m-1) !}(s-t)^{m-1} \nu^{(m)}(s) d s
$$

we have, for $t<a$,

$$
\begin{aligned}
(a-t)^{-m}|\nu(t)| & \leq(a-t)^{-1} \int_{t}^{a}\left|\frac{s-t}{a-t}\right|^{m-1}\left|\nu^{(m)}(s)\right| d s \\
& \leq \theta(t) \equiv(a-t)^{-1} \int_{t}^{a}\left|\nu^{(m)}(s)\right| d s
\end{aligned}
$$

thus the left hand side of (3) is estimated above by

$$
\int_{0}^{a} g(t)(a-t)^{2 m} \theta(t)^{2} d t \leq \mu(a ; g)^{2 m} \int_{0}^{a} \theta(t)^{2} d t .
$$

Therefore the inequality (3) follows from the estimate

$$
\int_{-\infty}^{a} \theta(t)^{2} d t \leq 2^{2} \int_{-\infty}^{a}\left|\nu^{(m)}(t)\right|^{2} d t
$$

which holds by the Hardy's inequality (Theorem 327 of Hardy, Littlewood and Polya [3]).

Q.E.D.

Second, in order to prove Lemma 2 below, we use the following one-dimensional interpolation inequality (see, e.g., Adams [1], the proofs of Lemmas 4.10 and 4.12). 
Proposition. There exist a constant $C_{0}$ and a positive integer $l$ such that, if $I$ is an (open) interval in $\mathbb{R}$ with the length $|I| \leq \delta$, then the inequality

$$
\int_{I}\left|u^{(k)}(t)\right|^{2} d t \leq C_{0}|I|^{-2 l}\left[\rho \int_{I}\left|u^{(m)}(t)\right|^{2} d t+\rho^{-k /(m-k)} \int_{I}|u(t)|^{2} d t\right]
$$

holds for $u \in C^{m}(I), 0 \leq k \leq m-1$ and $0<\rho \leq 1$.

Let $\phi$ be a function belonging to $C^{\infty}(\boldsymbol{R})$ such that

$$
0 \leq \phi \leq 1, \phi(\tau)=1 \text { for } \tau \leq 1, \text { and } \phi(\tau)=0 \text { for } \tau \geq 2 \text {. }
$$

Putting

$$
\chi(t)=\phi\left(\xi^{\gamma} f(t)\right), \quad \text { where } r=m /(\operatorname{lm}+m+1),
$$

we obtain

Lemma 2. There exists a constant $C$ such that the estimate

$$
\int\left|[\chi(t) \nu(t)]^{(m)}\right|^{2} d t \leq C\left[\int\left|\nu^{(m)}(t)\right|^{2} d t+\xi^{2 m} \int f(t)|\nu(t)|^{2} d t\right]
$$

holds for $\xi \geq 1$ and $\nu \in C_{0}^{m}(-\delta, \delta)$.

Proof. We have by the Leibniz rule

$$
\left|(\chi \nu)^{(m)}\right|^{2} \leq C_{1}\left[\left|\nu^{(m)}\right|^{2}+\sum_{j=1}^{m}\left|\chi^{(j)} \nu^{(m-j)}\right|^{2}\right],
$$

where $C_{1}$ depends only on $m$. Notice that, if $1 \leq j \leq m$, the function $\chi^{(j)} y^{(m-j)}$ vanishes on the outside of the open set

$$
I(\xi)=\left\{t \in(-\delta, \delta) ; \xi^{\gamma} f(t)>1\right\},
$$

and $I(\xi)$ is disjoint union of (at most) countably many open intervals $I_{p}=\left(\alpha_{p}, \beta_{p}\right)$ (contained in $(0, \delta)$ or $(-\delta, 0)$ ) with $\xi^{\gamma} f\left(\alpha_{p}\right)=1$ or $\xi^{\gamma} f\left(\beta_{p}\right)=1$. Since $\phi^{(k)}(\tau)$ $(k>0)$ vanishes to infinite order at $\tau=1$, we have $\left|\phi^{(k)}(\tau)\right| \leq C_{2}|\tau-1|^{l}$ for $\tau \geq 1$ and $1 \leqq k \leqq m$, where $C_{2}$ is independent of $\tau$ and $k$. Therefore, letting $\eta_{p}$ be $\alpha_{p}$ or $\beta_{p}$ with $\xi^{\gamma} f\left(\eta_{p}\right)=1$, we have, for $t \in I_{p}$ and $1 \leq j \leq m$,

$$
\begin{aligned}
\left|\chi^{(j)}(t)\right| & \leq \text { const. } \xi^{\gamma j} \max _{1 \leq k \leq m}\left|\phi^{(k)}\left(\xi^{\gamma} f(t)\right)\right| \\
& \leq \text { const. } \xi^{\gamma j} C_{2}\left|\xi^{\gamma} f(t)-\xi^{\gamma} f\left(\eta_{p}\right)\right|^{l} \\
& \leq C_{3} \xi^{\gamma j+\gamma l}\left|t-\eta_{p}\right|^{l} \leq C_{3} \xi^{\gamma(j+l)}\left|I_{p}\right|^{l}
\end{aligned}
$$

with a constant $C_{3}$ independent of $\xi, p$ and $t$. Hence 


$$
\int\left|\chi^{(j)^{(m-j)}}\right|^{2} d t \leq C_{3}^{2} \xi^{2 \gamma(j+l)} \sum_{p}\left|I_{p}\right|^{2 l} \int_{I_{p}}\left|\nu^{(m-j)}\right|^{2} d t .
$$

The above Proposition and (7) imply that the right hand side of the last inequality is estimated above by

$$
C_{3}^{2} \xi^{2 \gamma(j+l)} C_{0}\left[\rho \int_{I(\xi)}\left|\nu^{(m)}\right|^{2} d t+\rho^{-(m-j) / j} \xi^{\gamma} \int_{I(\xi)} f|\nu|^{2} d t\right]
$$

for $\rho \in(0,1]$. Putting $\rho=\xi^{-2 \gamma(j+l)}$, we obtain (5) by the definition of $\gamma$ and (6).

Q.E.D.

Now we can prove (2). Let $a=a(\xi)=\sup \left\{t \in(0, \delta) ; \xi^{\gamma} f(t) \leq 2\right\},-b=$ $-b(\xi)=\inf \left\{t \in(-\delta, 0) ; \xi^{\gamma} f(t) \leq 2\right\}$, where $r$ is the number as in (4). Then, $a$ and $b$ tend to 0 as $\xi \rightarrow \infty$, and, since $\xi=(2 / f(a))^{1 / \gamma}=(2 / f(-b))^{1 / \gamma},(1)$ implies that for every $\varepsilon>0$ there exists an $N=N_{\varepsilon}>1$ such that

$$
4\left[\mu(-b ; g)^{2 m}+\mu(a ; g)^{2 m}\right](\log \xi)^{2 m} \leq \varepsilon \quad \text { for } \xi \geq N .
$$

For arbitrary $\nu \in C_{0}^{m}(-\delta, \delta)$, we put $\nu_{1}=\chi \nu$ and $\nu_{2}=(1-\chi) \nu$, where $\chi$ is the function defined by (4). Since the support of $\nu_{1}$ is contained in $[-b, a]$, we can apply Lemma 1 to $\nu_{1}$. Hence

$$
\begin{aligned}
& (\log \xi)^{2 m} \int g(t)\left|\nu_{1}(t)\right|^{2} d t \leq \varepsilon \int\left|\nu_{1}^{(m)}(t)\right|^{2} d t \\
& \quad \leq \varepsilon C\left[\int\left|\nu^{(m)}(t)\right|^{2} d t+\xi^{2 m} \int f(t)|\nu(t)|^{2} d t\right]
\end{aligned}
$$

by Lemma 2. Since $g$ is bounded, $(\log \xi)^{2 m} g(t) \leq \varepsilon \xi^{2 m-\gamma}$ for $\xi \geq N$ provided $N$ is sufficiently large. Furthermore $\nu_{2}$ vanishes on the outside of the set $I(\xi)$ defined in (7), and, accordingly, the inequality $\left|\nu_{2}(t)\right| \leq|\nu(t)|$ yields the estimate

$$
(\log \xi)^{2 m} \int g(t)\left|\nu_{2}(t)\right|^{2} d t \leq \varepsilon \xi^{2 m} \int f(t)|\nu(t)|^{2} d t
$$

Since $|\nu(t)|^{2} \leq 2\left|\nu_{1}(t)\right|^{2}+2\left|\nu_{2}(t)\right|^{2}$, adding (8) and (9) ("sewing together"), we have (2).

Q.E.D.

\section{Proof of Theorem 2}

Let (C) be violated. We consider the case where $\mu(t ; g) \log f(t)$ does not converge to 0 (the other case is treated similarly). Then $f(0)=0$. There exist an $\varepsilon>0$ and sequences $s_{n}, t_{n}(n=1,2, \cdots)$ such that $s_{n}$ is between 0 and $t_{n}$,

$$
g\left(s_{n}\right)\left|t_{n}-s_{n}\right|^{2 m}\left|\log f\left(t_{n}\right)\right|^{2 m} \geq \varepsilon
$$

and $t_{n} \rightarrow 0$ as $n \rightarrow \infty$. 
We prove that $L$ becomes non-hypoelliptic by modifying the proof in [4] slightly. In fact, consider the eigenvalue problem in the interval $(-\delta, \delta)$

$$
\begin{aligned}
P(\xi) v(t) & \equiv\left[D_{t}^{2 m}+\xi^{2 m} f(t)\right] v(t)=\lambda g(t) v(t), \\
v^{(k)}(-\delta) & =v^{(k)}(\delta)=0, \quad k=0, \cdots, m-1,
\end{aligned}
$$

where $\xi$ is a real valued parameter. Here $\lambda$ is regarded as an eigenvalue. Let $\lambda(\xi)$ be the smallest positive eigenvalue and $v=v(t ; \xi)$ the corresponding eigenfunction such that $\|v(\circ ; \xi)\|^{2} \equiv \int_{-\delta}^{\delta}|v(t ; \xi)|^{2} d t=1$. Then we have

$$
\begin{aligned}
\lambda(\xi) & =(P(\xi) v(\circ ; \xi), v(\circ ; \xi)) /(g v(\circ ; \xi), v(\circ ; \xi)) \\
& =\inf \left\{(P(\xi) u, u) /(g u, u) ; u \in \mathbb{C}_{0}^{\infty}(-\delta, \delta), u \neq 0\right\}
\end{aligned}
$$

(the infimum of the Rayleigh ratio), where $(u, v)=\int_{-\delta}^{\delta} u(t) \overline{v(t)} d t$. Let $\xi_{n}$ be $f\left(t_{n}\right)^{-1 /(2 m)}$, which tends to $+\infty$ as $n \rightarrow \infty$, and let $J_{n}$ be the interval $\left(s_{n}, t_{n}\right)$ (or $\left.\left(t_{n}, s_{n}\right)\right)$. Then $\xi_{n}{ }^{2 m} f(t) \leq 1$ and $g(t) \geq g\left(s_{n}\right)$ for $t \in J_{n}$ by (A.2). Thus

$$
\begin{gathered}
\lambda\left(\xi_{n}\right) \leq g\left(s_{n}\right)^{-1} \cdot \inf \left\{\left[\left\|u^{(m)}\right\|^{2}+\|u\|^{2}\right] /\|u\|^{2} ; u \in C_{0}^{\infty}\left(J_{n}\right), u \neq 0\right\} \\
\quad \leq g\left(s_{n}\right)^{-1}\left[\text { const. }\left|t_{n}-s_{n}\right|^{-2 m}+1\right] \leq \text { const. }\left|\log f\left(t_{n}\right)\right|^{2 m}
\end{gathered}
$$

(for large $n$ ) by $(10)$. Hence $\lambda\left(\xi_{n}\right) \leq$ const. $\left(\log \xi_{n}\right)^{2 m}$. Let us put $\kappa_{n}=$ $\left[(-1)^{m+1} \lambda\left(\xi_{n}\right)\right]^{1 /(2 m)}\left(\operatorname{Re} \kappa_{n}>0\right)$. Then $\left|\kappa_{n}\right| \leq$ const. $\log \xi_{n}$. The rest of the proof is quite similar to that of [4], with the function $u_{n}$ in (2.8) of [4] replaced by $v\left(t ; \xi_{n}\right) \exp \left(i \xi_{n} x+\kappa_{n} y\right)$. Thus we omit it here.

Q.E.D.

\section{References}

[1] Adams, R.A., Sobolev Spaces, Academic Press, New York, 1975.

[2] Fedií, V.S., On a criterion for hypoellipticity, Math. USSR Sb., 14 (1971), 15-45.

[3] Hardy, G.H., Littlewood, J.E. and Pólya, G., Inequalities, 2nd ed., Cambridge University Press, Cambridge, 1952.

[4] Hoshiro, T., Hypoellipticity for infinitely degenerate elliptic and parabolic operators of second order, J. Math. Kyoto Univ., 28 (1988), 615-632.

[5] Hypoellipticity for infinitely degenerate elliptic and parabolic operators II, operators of higher order, J. Math. Kyoto Univ., 29 (1989), 497-513.

[6] Kusuoka, S. and Stroock, D., Applications of the Malliavin calculus, Part II, J. Fac. Sci. Univ. Tokyo Sect. IA, Math., 32 (1985), 1-76.

[7] Morimoto, Y., On the hypoellipticity for infinitely degenerate semi-elliptic operators, J. Math. Soc. Japan, 30 (1978), 327-358.

[ 8 ] - Non-hypoellipticity for degenerate elliptic operators, Publ. RIMS, Kyoto Univ., 22 (1986), 25-30.

[9] Criteria for hypoellipticity of differential operators, Publ. RIMS, Kyoto Univ., 22 (1986), 1129-1154.

[10] The uncertainty principle and hypoelliptic operators, Publ. RIMS, Kyoto Univ., 23 (1987), 955-964. 